\title{
Analysis of fungal bloodstream infection in intensive care units in the Meizhou region of China: species distribution and resistance and the risk factors for patient mortality
}

Guangwen Xiao ${ }^{1 *} \mathbb{D}$, Wanqing Liao ${ }^{2 *}$, Yuenong Zhang ${ }^{3}$, Xiaodong Luo ${ }^{1}$, Cailing Zhang ${ }^{4}$, Guodan Li', Yingping Yang ${ }^{1}$ and Yunyao $\mathrm{Xu}^{5}$

\begin{abstract}
Background: Fungal bloodstream infections (FBI) among intensive care unit (ICU) patients are increasing. Our objective was to characterize the fungal pathogens that cause bloodstream infections and determine the epidemiology and risk factors for patient mortality among ICU patients in Meizhou, China.
\end{abstract}

Methods: Eighty-one ICU patients with FBI during their stays were included in the study conducted from January 2008 to December 2017. Blood cultures were performed and the antimicrobial susceptibility profiles of the resulting isolates were determined. Logistic multiple regression and ROC curve analysis were used to assess the risk factors for mortality among the cases.

Results: The prevalence of FBI in ICU patients was $0.38 \%(81 / 21,098)$ with a mortality rate of $36 \%(29 / 81)$. Ninetyeight strains of bloodstream-infecting fungi, mainly Candida spp., were identified from these patients. Candida albicans was most common (43\%). Two strains of C. parapsilosis were no-sensitive to caspofungin, C. glabrata were less than $80 \%$ sensitive to azole drugs. Logistic multiple regression showed that age, serum albumin, APACHE II score, three or more underlying diseases, and length of stay in ICU were independent risk factors for mortality in FBI. ROC curve analysis showed that APACHE II scores $>19$ and serum albumin $\leq 25 \mathrm{~g} / \mathrm{L}$ were the best predictors of mortality.

Conclusion: Candida spp. predominated with high mortality rates among cases of FBI in ICU. Thus, clinical staff should enhance overall patient monitoring and concurrently monitor fungal susceptibility to reduce mortality rates.

Keywords: Intensive care unit, ICU, Fungal bloodstream infection, Epidemiology, Mortality risk factors

\footnotetext{
*Correspondence: 15916547823@163.com; liaowanqing@sohu.com

'Medical College, Jiaying University, Meizhou, People's Republic of China

${ }^{2}$ Shanghai Key Laboratory of Medical Fungal Molecular Biology, Shanghai, People's Republic of China

Full list of author information is available at the end of the article
}

C C The Author(s). 2020 Open Access This article is licensed under a Creative Commons Attribution 4.0 International License, which permits use, sharing, adaptation, distribution and reproduction in any medium or format, as long as you give appropriate credit to the original author(s) and the source, provide a link to the Creative Commons licence, and indicate if changes were made. The images or other third party material in this article are included in the article's Creative Commons licence, unless indicated otherwise in a credit line to the material. If material is not included in the article's Creative Commons licence and your intended use is not permitted by statutory regulation or exceeds the permitted use, you will need to obtain permission directly from the copyright holder. To view a copy of this licence, visit http://creativecommons.org/licenses/by/4.0/. The Creative Commons Public Domain Dedication waiver (http://creativecommons.org/publicdomain/zero/1.0/) applies to the data made available in this article, unless otherwise stated in a credit line to the data. 


\section{Background}

The incidence of fungal bloodstream infections caused by pathogens such as Candida spp. has increased in recent years, especially in intensive care units (ICUs) [1, 2]. Candida bloodstream infections have been reported to be the fourth highest in-hospital infection [3]. Studies have shown that the incidence of fungal bloodstream infections in ICUs is $0.22-4.1 \%$ in developing countries [4-7] and $0.024-0.687 \%$ in developed countries [8-10]. These rates appear to coincide with more widespread use of broad-spectrum antifungal drugs, glucocorticoids, and immunosuppressive agents, as well as the low immunity of ICU patients and use of central venous catheter technology and other invasive procedures; however, early symptoms of fungal bloodstream infection lack specificity, and low culture-positive rates can lead to a misdiagnosis [11]. Thus, hospitalization and timely, effective treatment might be delayed, increasing costs and mortality risks. In previous research reports, there were several studies on the pathogens and infection risk factors of fungal bloodstream infection and relatively few studies on the risk factors for the mortality of the patients.

Understanding species distribution, resistance, and mortality risk factors associated with pathogenic fungi and ICU fungal bloodstream infections is essential for improving our chances of an early diagnosis, early treatment, and a more positive prognosis. The aim of this study was to analyze the distribution, drug sensitivity, and mortality risk factors of ICU fungal bloodstream infections at three tertiary general hospitals in Meizhou, Guangdong Province, China, to provide baseline reference data for diagnosis and treatment of these infections.

\section{Methods}

\section{Patient selection}

Patients with new infections based on a primary disease who were admitted to ICU of three tertiary hospitals in the Meizhou area were included in the study conducted from January 2008 to December 2017. The patients had failed to respond to antibiotic treatment and tested positive by blood culture for yeast, yeast-like bacteria, and/or mold. We collected basic information on cases, including demographic data (sex, age), disease factors (diabetes, tumors, cardiovascular disease, COPD, digestive tract diseases, urinary system diseases, hypertension, and three or more combined underlying diseases), physiological indicators (serum albumin, serum urea nitrogen, $\gamma$--glutamyl peptidase, and APACHE II scores), invasive operational factors (e.g., tracheal intubation, multilumen catheter, central venous catheter, and catheter indwelling time), and treatment factors (e.g., length of ICU stay, gastrointestinal nutrition, emergency catheterization, blood transfusion, central venous catheter, patients in shock receiving catecholamines, and the combined use of antibiotics). The research protocol was approved by the ethics committee of the hospital.

\section{Culture and laboratory methods}

Blood samples that were taken and were cultured using the BACTEC FX400 Automated Blood Culture System (Becton Dickinson, Franklin Lakes, NJ, USA) and the BACTEC Plus/F resin aerobic culture bottle and fungal culture bottle (Becton Dickinson). The VITEK 2Compact microbial identification and analysis system and yeast biochemical card and analytical profile system systems (bioMérieux, Marcy-l'Étoile, France) were used to identify fungal organisms, using $C$. albicans ATCC 90028 and C. parapsilosis ATCC22019 as quality control strains.

ATB FUNGUS 3 fungal susceptibility reagent strips (bioMérieux) were used to determine antimicrobial susceptibilities to caspofungin, amphotericin B, fluconazole, voriconazole, and itraconazole on CHROMagar medium (Zhengzhou Biocell, Henan Province, China), the results were judged according to the current standards of specification.

\section{Statistical analyses}

Data were analyzed using SPSS 21.0 (IBM Corp., Waltham, NY, USA). Variables that displayed a normal distribution were expressed as the mean \pm standard deviation (SD) using one-way analysis of variance. Counts were expressed as the number of cases and their percentage rate and significance was measured using the chi-squared test.

Variables that were significant risk factors or close to statistical significance $(P<0.1)$ using univariate analysis were included in a two-class logistic multiple regression model for multivariate survival analysis. The best predictive values of independent risk factors for mortality according to related physiological indicators were analyzed using the ROC, where $P<0.05$ was considered statistically significant.

\section{Results}

Prevalence of intensive care unit bloodstream infection and fungal bloodstream infection from 2008 to 2017

The overall prevalence of ICU bloodstream infections was $6.54 \%(1380 / 21,098)$, the highest of which was $7.66 \%$ in 2011 and the lowest of which was $5.48 \%$ in 2013. The prevalence of fungal bloodstream infections was $0.38 \%(81 / 21,098)$, the highest of which was in 2016 at $0.47 \%$ and the lowest of which was in 2008 at $0.26 \%$ (Table 1). 
Table 1 Prevalence of intensive care unit (ICU) fungal bloodstream infection by year from 2008 to 2017

\begin{tabular}{llll}
\hline Year & Total blood culture $(n)$ & Positive result $(n / \%)$ & Fungal positive $(n / \%)$ \\
\hline 2008 & 1557 & $112(7.20 \%)$ & $4(0.26 \%)$ \\
2009 & 1571 & $109(6.94 \%)$ & $5(0.32 \%)$ \\
2010 & 1746 & $113(6.47 \%)$ & $6(0.34 \%)$ \\
2011 & 1672 & $128(7.66 \%)$ & $6(0.36 \%)$ \\
2012 & 1914 & $121(6.32 \%)$ & $8(0.42 \%)$ \\
2013 & 2135 & $117(5.48 \%)$ & $7(0.33 \%)$ \\
2014 & 2301 & $158(6.87 \%)$ & $10(0.43 \%)$ \\
2015 & 2503 & $147(5.87 \%)$ & $10(0.40 \%)$ \\
2016 & 2740 & $190(6.93 \%)$ & $13(0.47 \%)$ \\
2017 & 2959 & $185(6.25 \%)$ & $12(0.41 \%)$ \\
Total & 21,098 & $1380(6.54 \%)$ & $81(0.38 \%)$ \\
\hline
\end{tabular}

\section{Distribution of fungal strains}

Over the 10-year study period, 98 fungal strains from bloodstream infections, mainly Candida spp., were obtained from the blood cultures from 81 ICU patients. Candida albicans was most common (42/98, 43\%), followed by C. tropicalis (18/98, 18\%), C. glabrata (12/98, 12\%), C. parapsilosis (9/98, 9\%), C. krusei (5/98, 5\%), and C. guilliermondii (3/98, 3\%). Over the 10 -year study period, the prevalence of C. albicans decreased, while other Candida spp. increased each year (Fig. 1). In addition, three strains of Cryptococcus neoformans, two of Talaromyces marneffei, three of Pichia pastoris, and one of Saccharomyces cerevisiae were isolated.

\section{Antimicrobial susceptibility of strains}

The drug sensitivity analysis of the five main fungi in the 98 strains showed that $>95 \%$ of C. albicans strains were sensitive to all antifungal agents tested. All strains of $C$. tropicalis were sensitive to caspofungin and amphotericin B, and $>90 \%$ were sensitive to azoles. Candida glabrata were relatively less sensitive to azoles; $67 \%$ were sensitive to fluconazole and itraconazole, while all strains were sensitive to caspofungin and amphotericin B. Of the $C$. parapsilosis strains, $83 \%$ were sensitive to caspofungin, and all were sensitive to the other antifungals (Table 2).

\section{Risk factor analyses}

Twenty-nine of the 81 patients with fungal bloodstream infections in ICU died-a mortality rate of $36 \%$. Univariate analysis of mortality risk factors showed that advanced age, diabetes, cardiovascular disease, lower serum albumin, elevated $\gamma$-gamma glutamyl peptidase, APACHE II score, three or more underlying diseases, catheter indwelling time, and ICU stay were associated with the death rates (all $P<0.05$, Table 3). Sex, malignant tumor, COPD, digestive tract

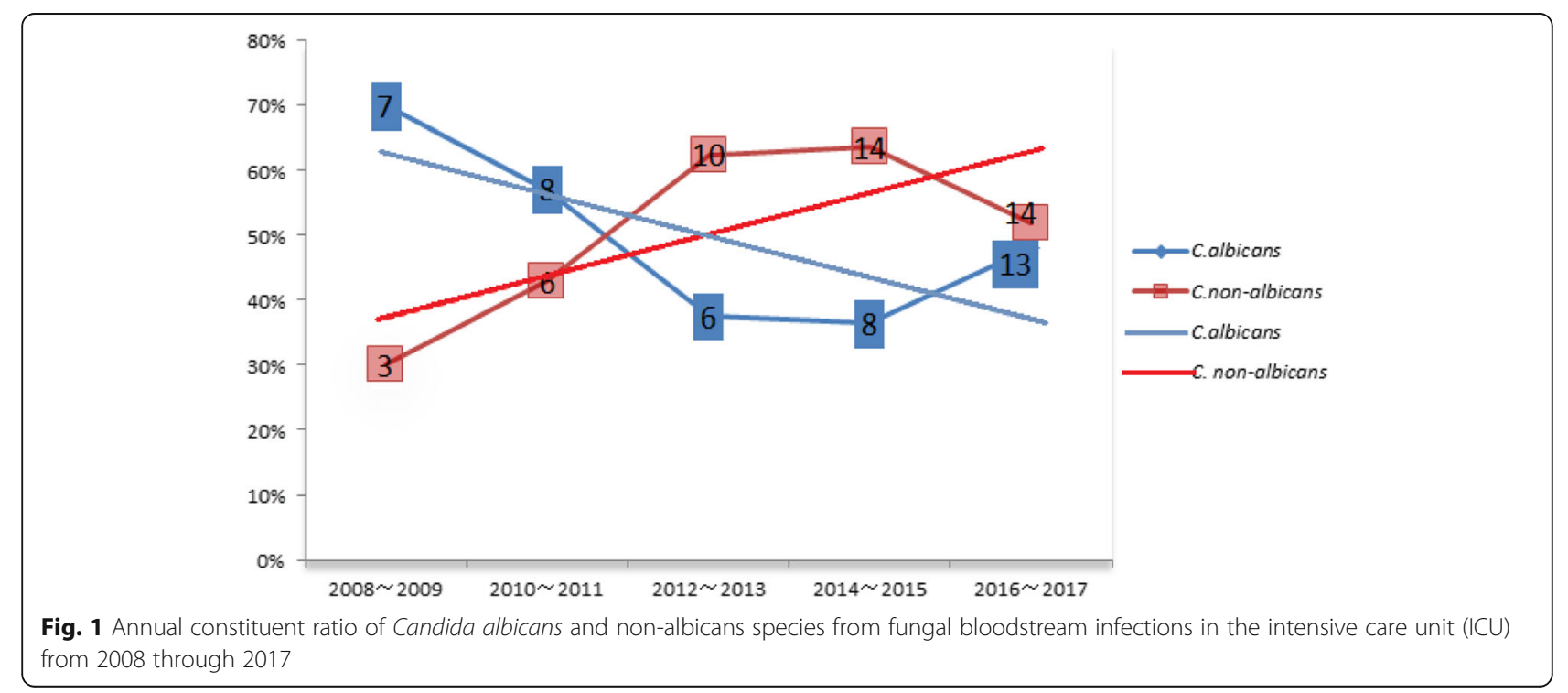


Table 2 Susceptibility rates of main fungi to five kinds of antifungal drugs (\%)

\begin{tabular}{|c|c|c|c|c|c|c|}
\hline \multirow[t]{2}{*}{ Fungal species } & \multirow{2}{*}{$\begin{array}{l}\text { Number of } \\
\text { strains (n) }\end{array}$} & \multicolumn{5}{|c|}{ Susceptibility rate (\%) } \\
\hline & & caspofungin & Amphotericin B & Fluconazole & Voriconazole & Itraconazole \\
\hline Candida albicans & 42 & 100 & 97.6 & 95.2 & 97.6 & 100 \\
\hline Candida tropicalis & 18 & 100 & 100 & 88.8 & 94.4 & 94.4 \\
\hline Candida parapsilosis & 12 & 83.3 & 100 & 100 & 100 & 100 \\
\hline Candida glabrata & 9 & 100 & 100 & 66.6 & 77.7 & 66.6 \\
\hline
\end{tabular}

disease, urinary tract disease, hypertension, serum urea nitrogen, tracheal intubation, multilumen catheter, central venous catheter, gastrointestinal nutrition, emergency catheterization, blood transfusion, patients in shock receiving cathecholamines, etc., and combined use of antibiotics were found not to be associated with death from fungal bloodstream infections (Table 3).
Following multivariate logistic regression, age, low serum albumin, APACHE II score, three or more combined underlying diseases, and ICU time were independently associated with mortality during hospitalization (Table 4).

ROC analysis of APACHE II score and serum albumin APACHE II score and serum albumin were the factors most strongly associated with mortality among patients

Table 3 Univariate analysis of mortality risk factors in patients in the intensive care unit with fungal bloodstream infections

\begin{tabular}{|c|c|c|c|c|}
\hline Variable & Survival $(n=52)$ & Death $(n=29)$ & $x^{2} / F$ & $P$ \\
\hline Sex, male/female $(n)$ & $32 / 20$ & $18 / 11$ & 0.002 & 0.962 \\
\hline Age (years; mean \pm s.d.) & $56.9 \pm 10.4$ & $65.7 \pm 11.8$ & 10.619 & 0.001 \\
\hline \multicolumn{5}{|l|}{ Disease factors } \\
\hline Diabetes $(n)$ & 10 & 12 & 4.616 & 0.032 \\
\hline Malignant tumor $(n)$ & 3 & 4 & 1.518 & 0.218 \\
\hline Cardiovascular disease $(n)$ & 8 & 16 & 14.135 & 0.000 \\
\hline Chronic obstructive pulmonary disease (COPD) $(n)$ & 5 & 4 & 0.329 & 0.566 \\
\hline Digestive tract disease $(n)$ & 5 & 4 & 0.329 & 0.566 \\
\hline Urinary system disease $(n)$ & 8 & 6 & 0.366 & 0.545 \\
\hline Hypertension ( $n$ ) & 13 & 13 & 3.358 & 0.067 \\
\hline Serum albumin $(g / L)$ & $39.5 \pm 8.6$ & $33.1 \pm 10.7$ & 7.91 & 0.005 \\
\hline Serum urea nitrogen $(\mu \mathrm{mol} / \mathrm{L})$ & $19.1 \pm 27$ & $30.7 \pm 53.2$ & 1.693 & 0.193 \\
\hline Y-gamma glutamyl peptidase $(U / L)$ & $36.2 \pm 24.7$ & $63.8 \pm 67.6$ & 6.629 & 0.010 \\
\hline Acute Physiology and Chronic Health Evaluation (APACHE) II score & $16.32 \pm 1.74$ & $19.97 \pm 2.90$ & 31.565 & 0.000 \\
\hline Merger of three or more underlying diseases $(n)$ & 6 & 12 & 7.82 & 0.005 \\
\hline \multicolumn{5}{|l|}{ Invasive operative factors } \\
\hline Tracheal intubation $(n)$ & 16 & 13 & 1.601 & 0.206 \\
\hline Multi cavity catheter $(n)$ & 11 & 11 & 2.649 & 0.104 \\
\hline Central venous catheterization ( $n$ ) & 23 & 16 & 0.893 & 0.345 \\
\hline Catheter retention time (d) & $12.0 \pm 4.0$ & $14.6 \pm 5.8$ & 5.070 & 0.024 \\
\hline \multicolumn{5}{|l|}{ Therapeutic factors } \\
\hline Hospitalization time in ICU (d) & $9.7 \pm 3.8$ & $12.1 \pm 6.7$ & 4.401 & 0.036 \\
\hline Gastrointestinal nutrition ( $n$ ) & 21 & 14 & 0.472 & 0.492 \\
\hline Emergency tube $(n)$ & 18 & 14 & 1.454 & 0.228 \\
\hline Blood transfusion ( $n$ ) & 17 & 12 & 0.611 & 0.434 \\
\hline Central venous catheter $(n)$ & 33 & 20 & 0.249 & 0.804 \\
\hline Patients in shock receiving cathecholamines etc. ( $n$ ) & 20 & 15 & 1.334 & 0.350 \\
\hline Combined use of antibiotics ( $n$ ) & 25 & 18 & 1.464 & 0.254 \\
\hline
\end{tabular}


Table 4 Logistic multiple regression analysis of mortality risk factors in patients in the intensive care unit (ICU) with fungal bloodstream infections

\begin{tabular}{lllll}
\hline Variable & Wald $x^{2}$ & $P$ & OR & $95 \% \mathrm{Cl}$ \\
\hline Age & 4.380 & 0.036 & 1.218 & $1.013-1.465$ \\
Diabetes & 3.106 & 0.078 & 19.544 & $0.717-533.069$ \\
Cardiovascular disease & 0.079 & 0.779 & 0.698 & $0.057-8.610$ \\
Hypertension & 0.882 & 0.346 & 6.494 & $0.131-322.387$ \\
Serum albumin & 6.679 & 0.010 & 0.639 & $0.455-0.897$ \\
Y-gamma glutamyl peptidase & 2.254 & 0.133 & 1.047 & $0.986-1.112$ \\
Acute Physiology and Chronic Health Evaluation (APACHE) II score & 8.163 & 0.004 & 6.330 & $1.821-22.001$ \\
Three or more underlying diseases & 4.560 & 0.030 & 0.003 & $0.000-0.576$ \\
Catheter retention time & 2.607 & 0.064 & 1.307 & $0.984-1.737$ \\
Hospitalization time in ICU & 4.052 & 0.035 & 1.322 & $1.020-1.714$ \\
\hline
\end{tabular}

Wald $X^{2}$, chi-squared test value; $O R$ odds ratio, $C l$ confidence interval

with fungal bloodstream infections. After subjecting these variables to ROC analysis, the results showed that when the APACHE II score was $>19$, the area under the curve (AUC) was 0.801 , and when serum albumin was $\leq 25 \mathrm{~g} / \mathrm{L}$, AUC was 0.636, which indicated that these were the best predictors for mortality among fungal bloodstream infection patients (Fig. 2).

\section{Discussion}

In agreement with our results, previous studies have also shown that the incidence of fungal bloodstream infection and the positive rate of blood culture are increasing each year. This may be closely related to the increasing use of broad-spectrum antibiotics, glucocorticoids, immunosuppressants, radiation therapy, chemotherapy, organ transplantation, catheter surgery, and the emergence of AIDS in recent years $[5,6,9,10]$.

Although the management of invasive fungal infections has made considerable progress in recent years, the prevalence of these infections continues to significantly increase, which warrants close attention from medical care providers. The incidence rate of fungal bloodstream infections in ICU patients in developing countries is 4 to 15 times higher than that in developed countries [11]. In this study, the prevalence of fungal bloodstream infections in ICUs in the three hospitals surveyed in Meizhou

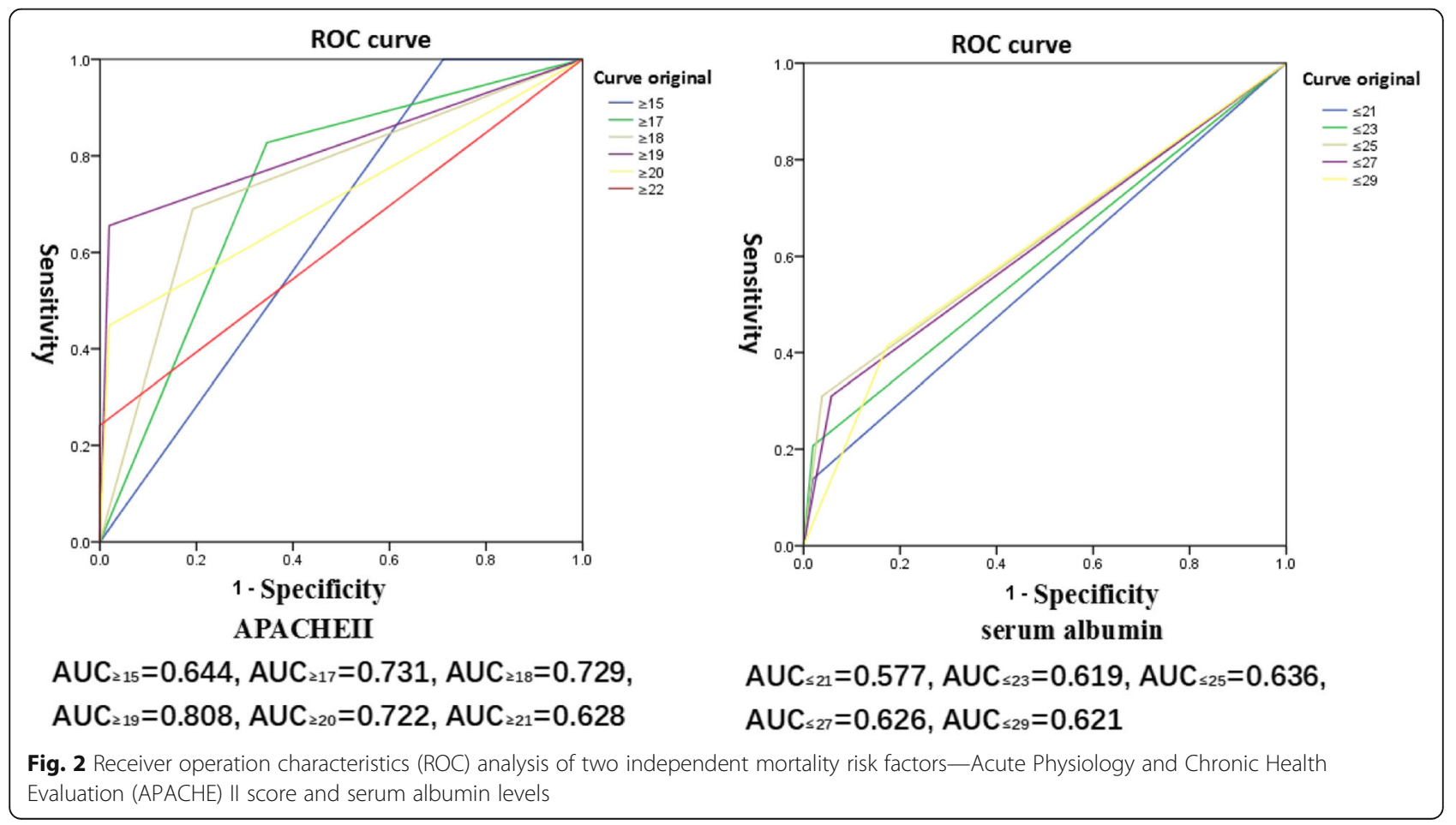


from 2008 through 2017 was $0.38 \%$. Compared with fungal bloodstream infections in ICU in developing countries, where the prevalence ranges from 0.22 to $4.1 \%$ [47], those in Meizhou were low and more comparable to the prevalence among developed countries, where the rates are reported to be $0.024-0.687 \%$ [8-10].

Candida albicans were more sensitive to azole drugs, such as fluconazole, than other Candida spp. Because azole drugs are those most commonly used for clinical fungal treatment, this might explain the increase in the proportion of non-albicans infections. Candida tropicalis has a high infection rate in tropical Asia [7, 12]. The Meizhou area is located in the southern part of China and has a subtropical climate, which might contribute to the high rate of ICU fungal infections in that area. Other reports show that $C$. parapsilosis is frequently carried on the hands of medical staff and has a tendency to form a biofilm on medical devices, which might explain its increasing prevalence in the area $[13,14]$.

In this study, nine patients were also infected with $C$. neoformans, T. marneffei, P. pastoris, and S. cerevisiae. Although the pathogenic ability of $C$. neoformans and $T$. marneffei was strong, that of $P$. pastoris and $S$. cerevisiae was weak. Pichia pastoris is used mainly as a research vector and is rarely isolated from human blood.

Most guidelines recommend the use of echinomycin for antifungal infections [15]; however, fluconazole is the most commonly used antifungal drug, and although it has a high mortality rate when used to treat candidemia, it is still widely used in developing countries [16]. In an Indian multicenter study of ICU-acquired candidemia, $64 \%$ of the patients were treated with fluconazole [17]. In this study, although most Candida spp. were more sensitive to azole drugs, the susceptibility of $C$. glabrata to azole drugs was relatively low, which suggests that testing for antimicrobial susceptibility is necessary for the correct selection of antifungal drugs.

Age is a significant risk factor for nosocomial infections [18], particularly fungal infections of the bloodstream [19, 20]. Candida parapsilosis more frequently infects younger populations, while C. glabrata and C. tropicalis are more common among the elderly [13]. In this study, age was an independent risk factor for infection, and the mortality risk increased with age (Table 4) for several reasons. First, older patients are more likely to have underlying diseases, low immunity, and decreased organ function, which would make them more susceptible to fungal bloodstream infections. Second, older patients might be given fewer antifungal treatments than younger patients, the practice of which is independently associated with a poor prognosis [21]. When the patient's condition deteriorates, the elderly patient or his or her guardian might decide to stop treatment if the patient's chance of recovery is low, the costs are higher if treatment is continued, or the patient's medical insurance does not cover the antifungal drug. Poor care of elderly patients by society and family members, as well as the relatively lagging development of the medical insurance industry, are important problems that affect the health of the elderly in China [22]. To effectively reduce the high mortality rate for elderly patients with fungal disease, measures should be taken to resolve the above-mentioned problems and ensure that antifungal treatment is administered.

Studies have shown that diabetes, tumors, neutropenia, and chronic renal insufficiency are risk factors for fungal bloodstream infections [23, 24]. In this study, diabetes, cardiovascular disease, and three or more underlying diseases were strongly associated with mortality from the infection (Table 3). Logistic multiple regression analysis showed that three or more underlying diseases were among the independent risk factors (Table 4), which might be associated with patients with multiple longterm underlying diseases and many iatrogenic invasive procedures. Treatments such as recent surgery, solid organ transplantation, hemodialysis, longer ICU stay ( $\geq 7$ d), mechanical ventilation, use of cardiovascular catheters, total parenteral nutrition, and catheters are other suggested risk factors for fungal bloodstream infections $[25,26]$. We found that length of stay in the ICU was also an important risk factor for mortality.

The APACHE II score is an important system used to determine the severity of a disease and estimates of mortality and plays an important role in judging the prognosis of bloodstream infections [27]. The higher the APAC HE II score, the lower the patient's immune function; the higher the probability of infecting pathogens, such as fungi; and the higher the chance of death. Serum albumin levels are also important factors that affect bloodstream infections in hospitals [28-30] and are associated with increased mortality from candidemia [12]. Low serum albumin levels affect the body's immune function, including barrier function, leukocyte phagocytosis, and complement function, resulting in prolonged infection time, anti-infective effects, and increased mortality. Hypoalbuminemia can lead to serious complications, such as sepsis and septic shock [28, 29]. The results of this study showed that the APACHE II score and serum albumin levels were two important independent mortality risk factors in ICU fungal infections (Table 4). According to our findings, we recommend that patients with APACHE II scores $>19$ and serum albumin $<25 \mathrm{~g} / \mathrm{L}$ should receive immediate clinical attention and increased vigilance for possible cases of fungal bloodstream infections.

Our study had several limitations. First, the design of the study limited our access to information on the use of antifungal drugs before hospitalization, and these data 
could be important for analyzing the emergence of antifungal resistance of the non-albicans species, for example, C. glabrata. Second, we analyzed only the presence or absence of exposure to certain risk factors for patient death instead of the duration of exposure. Because the study was not designed to quantify exposure time and the variable was not available for analysis, its associated bias could not be determined. Third, whether patients with fungal bloodstream infections carry AIDS and its related indicators is also an important factor in the death of patients, but in this study, only 2 cases (all deaths) were carried out with AIDS, and fewer cases were not included on analysis of the risk factors for patient mortality. Analysis of risk factors, which may be quite different from studies in other regions. In addition, because of differences in the epidemiological characteristics of antifungal use and candidemia in different countries and regions, we might not be able to extend some of our conclusions to other countries and regions, and additional research is needed in different geographical regions.

\section{Conclusions}

Candida albicans, C. tropicalis, and C. parapsilosis were found to be the most common pathogens that cause fungal bloodstream infections in ICU patients in Meizhou, Guangdong, China. The common fungi in this area are highly sensitive to common antifungal drugs, but attention should be paid to the low sensitivity of C. glabrata to azole drugs and the resistance of $C$. parapsilosis to caspofungin. In addition, advanced age, serum albumin, APACHE II score, three or more combined underlying diseases, and ICU stay were independent risk factors for fungal bloodstream infections, each of which must be highly assessed in clinical practice. For high-risk groups, emphasis should also be placed on patient monitoring and risks of infection.

\section{Abbreviations}

APACHE II: Acute physiology and chronic health evaluation II; ROC: Receiver operating characteristics; COPD: Chronic obstructive pulmonary disease

\section{Acknowledgements}

Not Applicable.

\section{Authors' contributions}

$\mathrm{G}$ Xiao planned the study, wrote the protocol, analyzed the data, and wrote the report. W Liao gave guidance on the design of the study and revised the manuscript. $Y$ Zhang, $C$ Zhang and $Y X u$ were involved in collecting. $X$ Luo, $G \mathrm{Li}$ and $Y$ Yang were involved in analyzing data and translating. All authors read and approved the final manuscript.

\section{Funding}

This work was supported by the Shanghai Key Laboratory of Medical Fungal Molecular Biology Open Project Foundation (Grant 14DZ2272900) to G Xiao. The funders played no role in study design, data collection and analysis, decision to publish, or preparation of the manuscript. None of the other authors have any financial disclosures.

\section{Availability of data and materials}

The datasets used and/or analyzed during the current study are available from the corresponding author on reasonable request.

\section{Ethics approval and consent to participate}

The study was approved by the local institutional review board of the Medical College, Jiaying University; informed consent was not required from patients because of the observational nature of the surveillance.

\section{Consent for publication \\ Not applicable.}

\section{Competing interests}

The authors declare that they have no competing interests.

\section{Author details}

'Medical College, Jiaying University, Meizhou, People's Republic of China. ${ }^{2}$ Shanghai Key Laboratory of Medical Fungal Molecular Biology, Shanghai, People's Republic of China. ${ }^{3}$ The First Department of Anesthesiology, People's Hospital of Meizhou, Meizhou, People's Republic of China.

${ }^{4}$ Department of Anesthesiology, Chinese Medical Hospital of Meizhou, Meizhou, People's Republic of China. ${ }^{5}$ Department of Cardiology, Yuedong Hospital the Third Affiliated Hospital of Sun Yat-Sen University, Meizhou, People's Republic of China.

Received: 25 July 2019 Accepted: 26 July 2020

Published online: 14 August 2020

\section{References}

1. Aguirre-Avalos G, Mijangos-Mendez JC, Zavala-Silva ML, Coronado-Magana H, Amaya-Tapia G. Bacteremia caused by Acinetobacter baumannii among patients in critical care. Gac Med Mex. 2009;145:21-5.

2. Gudlaugsson O, Gillespie S, Lee K, Vande BJ, Hu J, Messer S, et al. Attributable mortality of nosocomial Candidemia, Revisited. Clin Infect Dis. 2003;37:1172-7.

3. Pfaller MA, Diekema DJ, Jones RN, Sader HS, Fluit AC, Hollis RJ, et al. International surveillance of blood stream infect ions due to Candida species:frequency of occurrence and in vitro susceptibilities to fluconazole, ravuconazole, and voriconazole of isolates collected from 1997 through 1999 in the SENTY antimicrobial surveillance program. J Clin Microbiol. 2001;39(suppl. 9):3254-9.

4. Yapar N. Epidemiology and risk factors for invasive candidiasis. Ther Clin Risk Manag. 2014;10:95-105.

5. Chapman B, Slavin M, Marriott D, Halliday C, Kidd S, Arthur I, et al. Changing epidemiology of candidaemia in Australia. J Antimicrob Chemother. 2017; 72:1103-8.

6. Asmundsdottir LR, Erlendsdottir $H$, Gottfredsson M. Nationwide study of candidemia, antifungal use, and antifungal drug resistance in Iceland, 2000 to 2011. J Clin Microbiol. 2013;51:841-8.

7. Kreusch A, Karstaedt AS. Candidemia among adults in Soweto, South Africa, 1990-2007. Int J Infect Dis. 2013;17:e621-3.

8. Poowanawittayakom N, Dutta A, Stock S, Touray S, Levitz SM. Reemergence of intravenous drug use as risk factor for candidemia, massachusetts, usa. Emerg Infect Dis. 2018;24(4):631-7.

9. Khan Z, Ahmad S, Al-Sweih N, Joseph L, Alfouzan W, Asadzadeh M. Increasing prevalence, molecular characterization and antifungal drug susceptibility of serial Candida auris isolates in Kuwait. PLoS One. 2018; 13(suppl. 4):e0195743.

10. Tan BH, Chakrabarti A, Li RY, Patel AK, Watcharananan SP, Liu Z, et al. Incidence and species distribution of candidaemia in Asia: a laboratorybased surveillance study. Clin Microbiol Infect. 2015;21:946-53.

11. Barchiesi F, Orsetti E, Gesuita R, Skrami E, Manso E, Candidemia SG. Epidemiology, clinical characteristics, and outcome of candidemia in a tertiary referral center in Italy from 2010 to 2014. Infection. 2016;44:205-13.

12. Chen LY, Kuo SC, Wu HS, Yang SP, Chan YJ, Chen LK, et al. Associated clinical characteristics of patients with candidemia among different Candida species. Microbiol Immunol Infect. 2013;46:463-8.

13. Barchiesi F, Caggiano G, Di Francesco LF, Montagna MT, Barbuti S, Scalise G. Outbreak of fungemia due to Candida parapsilosis in a pediatric oncology unit. Diagn Microbiol Infect Dis. 2004;49(suppl. 4):269-71. 
14. Sardi JCO, Scorzoni L, Bernardi T, Fusco-Almeida AM, Mendes Giannini MJ. Candida species: current epidemiology, pathogenicity, biofilm formation, natural antifungal products and new therapeutic options. J Med Microbiol. 2013;62(suppl. 1):10-24.

15. Pappas PG, Kauffman CA, Andes DR, et al. Clinical practice guideline for the Management of Candidiasis: 2016 update by the Infectious Diseases Society of America. Clin Infect Dis. 2015;62:e1-e50.

16. Nucci M, Thompson-Moya L, Guzman-Blanco M, Tiraboschi IN, Cortes JA, Echevarria J, et al. Recommendations for the management of candidemia in adults in Latin America. Rev Iberoam Micol. 2013;30:179-88.

17. Chakrabarti A, Sood P, Rudramurthy SM, Chen S, Kaur H, Capoor M, et al. Incidence, characteristics and outcome of ICU-acquired candidemia in India. Intensive Care Med. 2015;41:285-95.

18. Aguirre-Avalos G, Mijangos-Méndez JC, Zavala-Silva ML, Coronado-Magana H, Amaya-Tapia G. Bacteremia caused by Acinetobacter baumannii among patients in critical care. Gac Med Mex. 2009;145(suppl. 1):21-5.

19. Pfaller MA, Andes DR, Diekema DJ, Horn DL, Reboli AC, Rotstein C, et al. Epidemiology and outcomes of invasive candidiasis due to non-albicans species of Candida in 2,496 patients: data from the prospective antifungal therapy (PATH) registry 2004-2008. PLoS One. 2014;9(suppl. 7):e101510.

20. Wisplinghoff H, Ebbers J, Geurtz L, Stefanik D, Major Y, Edmond MB, et al. Nosocomial bloodstreaminfections due to Candida spp. in the USA: species distribution, clinical features and antifungal susceptibilities. Int J Antimicrob Agents. 2014;43(suppl. 1):78-81.

21. Lee I, Fishman NO, Zaoutis TE, Morales KH, Weiner MG, Synnestvedt M, et al. Risk factors for fluconazole-resistant Candida glabrata bloodstream infections. Arch Intern Med. 2009;169:379-83.

22. Chen Z, Yu J, Song Y, Chui D. Aging Beijing: challenges and strategies of health care for the elderly. Ageing Res Rev. 2010;9(Suppl 1):S2-5.

23. Bassetti M, Trecarichi EM, Righi E, Sanguinetti M, Bisio F, Posteraro B, et al. Incidence, risk factors, and predictors of outcome of candidemia. Survey in 2 Italian university hospitals. Diagn Microbiol Infect Dis. 2007;58:325-31.

24. Arendrup MC. Epidemiology of invasive candidiasis. Curr Opin Crit Care. 2010;16:445-52.

25. Pfaller MA, Castanheira M. Nosocomial candidiasis: antifungal stewardship and the importance of rapid diagnosis. Med Mycol. 2016;54:1-22.

26. Bansal N, Gopalakrishnan R, Sethuraman N, Ramakrishnan N, Nambi P, Kumar $D$, et al. Experience with $\beta$-d-glucan assay in the management of critically ill patients with high risk of invasive candidiasis: an observational study. Indian J Crit Care Med. 2018;22(5):364.

27. Lissauer ME, Leekha S, Preas MA, Thom KA, Johnson SB. Risk factors for central line-associated bloodstream infections in the era of best practice. J Acute Care Surg. 2012;72:1174-80.

28. Lee JH, Kim J, Kim K, Jo YH, Rhee J, Kim TY, et al. Albumin and C-reactive protein have prognostic significance in patients with community-acquired neumonia. J Crit Carc. 2011;26:287-94.

29. Delgado-Rodriguez M, Medina-Cuadros M, Gomez-Ortega A, MartinezGallego G, Mariscal-Ortiz M, Martinez-Gonzalez MA, et al. Cholesterol and serum alhumin levels as predictors of cross infection, death and length of hospital stay. Arch Surg. 2002;137:805-12.

30. Artero A, Zaragoza R, Camarena J, Sancho S, Gonzalez R, Nogueira JM. Prognostic factors of mortality in patients with community-acquired bloodstream infection with severe sepsis and septic shock. J Crit Care. 2010; 25:276-81.

\section{Publisher's Note}

Springer Nature remains neutral with regard to jurisdictional claims in published maps and institutional affiliations.

\section{Ready to submit your research? Choose BMC and benefit from:}

- fast, convenient online submission

- thorough peer review by experienced researchers in your field

- rapid publication on acceptance

- support for research data, including large and complex data types

- gold Open Access which fosters wider collaboration and increased citations

- maximum visibility for your research: over $100 \mathrm{M}$ website views per year

At BMC, research is always in progress.

Learn more biomedcentral.com/submissions 